Relations industrielles

Industrial Relations

\title{
Techniques des relations de travail
}

\section{Gérard Tremblay}

Volume 4, numéro 8, avril 1949

URI : https://id.erudit.org/iderudit/1023511ar

DOI : https://doi.org/10.7202/1023511ar

Aller au sommaire du numéro

Éditeur(s)

Département des relations industrielles de l’Université Laval

ISSN

0034-379X (imprimé)

1703-8138 (numérique)

Découvrir la revue

Citer cet article

Tremblay, G. (1949). Techniques des relations de travail. Relations industrielles / Industrial Relations, 4(8), 71-72. https://doi.org/10.7202/1023511ar

Tous droits réservés @ Département des relations industrielles de l’Université Laval, 1949
Ce document est protégé par la loi sur le droit d'auteur. L’utilisation des services d'Érudit (y compris la reproduction) est assujettie à sa politique d'utilisation que vous pouvez consulter en ligne.

https://apropos.erudit.org/fr/usagers/politique-dutilisation/ 


\section{Bulletin \\ des \\ relations in \\ industrielles}

Volume 4, numéro 8

QUÉBEC

Avril 1949

Publié par le

Département des relations industrielles, Faculté des sciences, sociales, Université Laval.

Georges-Henri Lévesque, o.p., doỵen

Gérard Tremblay, directeur

Gerard Dion, sous-directeur

Jean Gagne, secrétaire

Charles BÉlanger, administrateut

Le Bulletin paraît mensuellement de septembre à juin (dix numéros par année). Abonnement annuel: Canada: $\$ 2.00$; étran-

ger: \$2.50. Vingt-cinq cents le numéro.

Publication répertoriée dans le "Canadian Index".

Adressez toute correspondance
au secrétaire de rédaction
GÉrARD Dion
2, rue de l'Université, Québec.

Bulletin des relations industrielles

\begin{tabular}{ll}
\hline Volume 4, numéro 8 & Avril 1949
\end{tabular}

Sommaire

Techniques des relations de travail

Gérard TrEMblay..

Application de la convention collec-

tive et administration du personnel

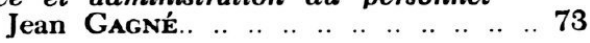

Conseil d'entreprise..

Mouvement des salaires dans le commerce de l'alimentation en gros de Québec

Raymond GagnÉ.. ..

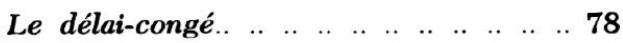

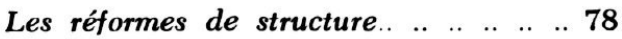

La procédure d'arbitrage.

79

\section{Collaborateurs}

Gagné, Jean, B.A., M.Sc.Soc., LL.L., secrétaire du Département des relations industrielles de l'Université Laval.

GaGné, Raymond, B.A., M.Sc.Soc., statisticien, Comité paritaire du commerce de l'alimentation en gros de Québec.

Tremblay, Gérard, L.Ph., Sous-ministre du travail, professeur, directeur du Département des relations industrielles de la Faculté des sciences sociales de l'Université Laval.

Autorisé comme envoi postal de deuxième classe, ministère des postes, Ottawa, Canada.
TECHNIQUES DES RELATIONS DE TRAVAIL

\author{
Gérard Tremblay
}

«Techniques des relations de travail 》 tel est le thème du quatrième Congrès du Départemerıt des relations industrielles. Ce thème découle naturellement de celui de l'an dernier: «Formes de collaboration patronale-ouvrière». L'une des formes reconnue et bien actuelle de cette collaboration est certes la négociation collective des conditions de travail qui donne naissance à la convention collective ellemême. Or, si nous avons étudié l'an passé la convention collective, forme éminente de collaboration patronale-ouvrière, ne convient-il pas d'en déceler maintenant les techniques? Techniques de la convention collective ou techniques des relations de travail n'est-ce pas, sur un plan général, les moyens de solution d'un même problème ?

Les relations de travail sont précisément ces rapports humains qui se développent forcément entre le capital et le travail dans le processus de la production et de la distribution des biens. Or la convention collective est aussi l'expression de rapports humains entre l'employeur et le syndicat de ses employés. Et il se trouve que le contenu de la convention indique aussi les normes que devront suivre les deux parties dans l'oeuvre de production et le partage de la plus-value ajoutée par leur apport commun à la matière inerte.

Ce parallélisme des définitions des relations de travail et de la convention collective n'a pas toujours été possible. Au siècle dernier, les relations de travail se sont presque toujours maintenues dans le cadre des relations individuelles. Mais l'évolution s'est faite et se continue de l'individuel au collectif.

Il y a aujourd'hui un régime juridique de la convention collective. Celle-ci est reconnue de bien commun par la loi dans tout le Canada comme du reste aux Etats-Unis et en Europe démocratique. Patrons et ouvriers n'en mettent plus en doute l'excellence, si même l'accord des deux groupes ne s'établit que dans la peine. Les relations de travail de plus en plus se ramènent donc à la convention collective, si bien que l'on puisse dire: donnez-moi de bonnes conventions collectives et je vous assure de bonnes relations de travail. 
Or ces bonnes relations de travail présupposent la mise en oeuvre de bonnes techniques. Et ces techniques ne sont pas seulement une savante codification de règles légales ou de coutumes. Elles sont d'abord humaines, car elles engagent tout l'homme: sa pensée, sa conscience, ses instincts, ses besoins, sa vie. Et il faut donc admettre que les techniques des relations de travail ont d'abord un aspect humain et moral qui est moins apparent mais qui dépasse toujours leur aspect scientifique et légal. Ce à quoi il faut tendre c'est à l'intégration dans l'intelligence et le coeur des hommes de ces multiples aspects d'un problème complexe et simple à la fois: le problème de l'homme dans ses rapports avec ses semblables en face des activités économiques.

On parlera au cours de ce congrès du cycle complet des relations de travail: organisation du syndicat ouvrier et du syndicat patronal. Les deux sont nécessaires. On ne peut établir de normes sociales dans un monde inorganisé et individualiste. C'est le régime de l'incohérence et du chaos. Un certain parallélisme doit se constater entre les organisations patronales et ouvrières. Il est illogique d'obliger un syndicat de métier à négocier avec une association patronale hétéroclite. Il y a donc une technique d'organisation qui doit tenir compte du particularisme des communautés de travail.

Ces organisations doivent, par leurs représentants, préparer et conclure des accords; elles doivent en appliquer les dispositions. Nous sommes ici au coeur même des relations de travail. Les négociations directes sont les meilleures; elles préviennent les recours dilatoires à la conciliation, à la médiation et à l'arbitrage. C'est dans la négociation que l'on juge des qualités du représentant syndical comme du délégué patronal. Qualités de coeur et d'intelligence. Droiture de la pensée, compréhension, loyauté, patience et fermeté; connaissance des éléments du débat, des techniques de l'industrie, des conditions du marché; dialectique persuasive et lumineuse. On ne manquera pas au cours de ces journées d'étude de mettre en lumière la nécessité urgente de développer chez les responsables ces qualités et bien d'autres.
Les procédures de conciliation, de médiation et d'arbitrage seront, je crois, toujours nécessaires bien qu'on puisse en réduire l'usage dans la mesure où les intéressés ont su développer leur sens de responsabilité. Aussi bien est-il opportun de s'arrêter aux techniques qui doivent les conditionner. La conciliation est une phase plus avancée de la négociation. Un tiers intervient pour en mieux diriger les débats pour suggérer la solution de compromis auquel on n'avait pas songé et qui permettra la sauvegarde des prestiges. Le conciliateur doit développer chez lui toutes les qualifications que l'on réclame du négociateur. De même le médiateur, qui se place sur un plan d'autorité morale plus élevé, devra lui aussi faire appel à toutes les ressources de la psychologie, de la dialectique et à un haut sens de l'équité s'il veut amener les parties à accepter ses propositions. La procédure d'arbitrage a aussi ses écueils non seulement pour les arbitres mais aussi pour les délégués des parties qui doivent participer aux débats. Circonspection dans le choix d'un président, netteté des propositions à débattre, comparution des témoins et des experts, préparation de la preuve et des factums, plaidoiries, voilà autant de sujets d'étude pour les techniciens des relations de travail.

Où doit nous conduire cet effort pour améliorer les techniques? Tout simplement à nous convaincre que d'abord la compétence ne s'improvise pas mais se prépare ardument; qu'il y a, à raison de l'industrialisation de la province et du développement des rapports collectifs entre patrons et ouvriers et aussi du fait d'une législation plus évoluée, un besoin de plus en plus pressant de bons spécialistes en relations industrielles qui doivent s'intégrer à un milieu social où ils pourront servir le bien commun en servant judicieusement un groupe.

De saines relations de travail aidées de techniques éprouvées permettront finalement d'assurer la dignité de lhomme au travail, de favoriser cette équitable distribution des richesses que recommande l'Eglise et que réclame le peuple et de procurer enfin à notre patrie une paix sociale qui soit oeuvre de justice et de charité. 